\title{
Robot Assisted Routing and Navigation System for Visually Impaired People
}

\author{
Vishnu Prasad S, T. Shanmuganantham
}

\begin{abstract}
This paper deals with the work on creating a robot assisted navigation system for the visually impaired people. The ability to see is an important gift that helps human beings to live their everyday lives, but not all people are blessed with this ability. The normal blind navigation devices deals with on-body devices that the user must carry, but this paper is talking about is a small rover that can be held by the user using a hanlde. The device will contain a raspberry pi module for taking data from a camera on-board to see the environment and make object and obstacle detection. The 4-WD rover will be controlled by an Arduino device which will be connected to the mobile of the user via a Bluetooth module. The user just has to say the name of the location he needs to go to by opening an application, the rest of the routing and transport will be done by the raspberry pi and Arduino.
\end{abstract}

Keywords: Arduino, Compass Sensor, Mobile application, Raspberry Pi

\section{INTRODUCTION}

$\mathrm{M}$ any studies have shown that the number of visually impaired people who live in this world have kept on increasing. The main difficulties that a visually impaired person face is the inability to know where he is, inability to read signs and to see where he is moving and also to move from location A to B in a completely unknown environment [1]. A person with perfect vision will find it difficult to traverse an unknown location for the first time then the situation of a visually impaired person is all the more difficult. The main thing any work in this field must do is to deciding what all information needs to be fed to the user and how it must be fed to him/her [2]. From the ancient times blind or visually impaired people relied on a long cane or a guide dog for moving from place to place. The cane served only one purpose, to identify if there is any obstacle is in front of him, the guide dog helped him in navigating a terrain without getting injured. So the work mentioned in this paper is that it combines the functions of the cane and the guide dog, it will help the user identify if there are any obstacles in

Revised Manuscript Received on February 05, 2020.

* Correspondence Author

Vishnu Prasad S*, Final year M.Tech Student, Department of Electronics Engineering, Pondicherry University, Pondicherry, India, vishnuprasads.ec@gmail.com

T. Shanmuganantham, Assistant Professor, Department of Electronics Engineering , Pondicherry University, Pondicherry, India, shanmugananthamster@gmail.com

(C) The Authors. Published by Blue Eyes Intelligence Engineering and Sciences Publication (BEIESP). This is an open access article under the CC BY-NC-ND license (http://creativecommons.org/licenses/by-nc-nd/4.0/) front of him, it will notify the user of any potential threats like tree branches or other things, it will navigate him to the destination and it will also notify him about the people he is encountering. The rest of the paper prepared according to the following: Section II describes some research related to the navigations techniques that exists today to help these people. Section III outlines the approach proposed. Section IV sets out the limitations of the experimental setup and future improvements needed to be worked on. The experimental findings and visual analysis have been discoursed in Section $\mathrm{V}$. The conclusion is given in section VI.

\section{RELATED WORKS}

There are two types of navigation techniques which developers use to help pinpoint the location and the path the person need to travel through, these are (a) Position Based Navigation, (b) Velocity Based Navigation. The position based devices uses the help of GPS sensors to find the position of the person before making routing. In the velocity based method of navigation the user's normal path is monitored and the time or distance the user takes or moves between two check points are stored in the data base. Then this data is later used to help them for moving between those locations at a later time [3], [4], [5]. The main problem with this thing was that the location of the user was not known, so if the initial location is not similar to the stored initial location then the entire routing can be disrupted causing the user injuries. The routing in the indoor and outdoor locations is done very differently thus it is very difficult to use the same device in both indoor and outdoor environment. The navigation techniques used in the outdoor devices are mostly GPS based, these use a GPS module to identify the location of the user and then route the user to his location [6-9]. Most devices that are used in the outdoor environment are "ONBODY" devices, that is, these devices must be kept on the body of the user/ he needs to carry it where ever he/she goes. The indoor devices can be on-body devices or they can be integrated with our smart-phones to reduce the weight of the device. The main problem with on-body devices are the discomfort that it causes to the wearer. These devices utilizes different methods for obstacle detection and routing, but the main problem with these devices are the limited resolution when it comes to the GPS sensor, i.e. in outdoor environment if the user is moving at the edge or side of the road the GPS reading will be same so the device will not warn him of his position with respect to the road. Then the models used in the indoor models must contain a map of the entire location, the position of each and every thing in that location and it must also need a method to identify the relative position of the user in a room.

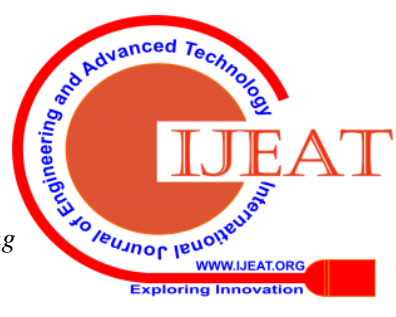




\section{Robot Assisted Routing and Navigation System for Visually Impaired People}

These devices usually use the received signal strength index from different transmitters on the walls to identify the relative position of the user in a room [10 - 13]. These devices will allow the user the freedom to move about the room as he likes but the user needs to be constantly updated about his location with respect to a check point in each room regularly. The other method is to use RF-ID tags or QR codes on the path that the user needs to travel through in the indoor location. This reduces his ability to move freely he/ she can only travel through the specified path [14]. There has been many initiatives that came up recently to help visually impaired people to come forward in different spheres of the society, for this to make them more comfortable devices have been made to help them move about in the urban areas[15], in museums [16], traffic areas [17], airports[18] etc. These devices will help them to have a better interaction with the environment and will also help them to move about different locations more easily, it helps them to identify what's in front of them and when the next bus to location is available at and at what place. Then there are devices that can be used in both indoor and outdoor environments [19]. The devices that have been mentioned till now all requires maintenance and updating there are societies and training organizations that provide such services [20]. The devices mentioned are all having either the routing or navigation functions or obstacle detection features embedded in them, but the dual functional devices are on-body devices, so these systems take a tradeoff between obstacle detection and user convenience. The work in this paper is having both obstacle detection and navigation and it also includes object identification and a facial recognition feature that allows the user to identify what type of obstacles are in front of him/her and will also tell them if a pre-saved person is in front of them.

\section{THE SYSTEM}

When a visually challenged person travels, he is not having access to many inputs like orientation, location, possible obstacles and building signs. So in most cases they will most probably travel through routes that are familiar to them. This work comes into existence so that these information are given to the user whenever and where ever he/ she needs it. The device in this paper has 3 parts, (a) 4 Wheel drive car (b) a mobile application and (c) the controllers. The routes that the user needs to go through is pre-saved in the memory, the 4WD car will be attached with a small handle that he user can hold, then he will use the mobile application and shake the mobile to activate the voice scanner, once the scanner is activated a beep sound will be heard after which the user can specify the location to which they need to go to. Once the destination is obtained then the data is given to the Arduino controller which will then use the saved routing table to identify the route and then route the user to the desired destination. The Arduino will control the car, and then the user can hold on to the handle of the car so that it will guide him/her to the destination just like a guide dog will, the handle will have a push button switch, the car moves only when it is pressed thus giving more convenience to the user. The ultrasonic sensor will be placed on the car itself so no discomfort comes to the user, the mobile camera will be used to connect with the Raspberry pi using IP camera application, thus there is no need for a special camera just the user needs to keep the mobile on the handle attached so the

user need not carry anything on his/her person. Then while he/she moves from A to B the location is constantly updated and the camera will monitor the obstacles and people in front of the user. The device will thus give accurate information about all the things happening in front of the user.

\section{A. Pre-requisites and routing}

The prerequisite that was done in the test environment was to identify the routine locations that the person travels to like his hostel, department, mess, library and the gate. So once the locations were identified RF modules were placed in some locations between these destinations and the magnetic declinations of these locations were identified. Then a routing table was made in which it shows which all RF locations should the user cross to reach from point $A$ to $B$ where $\mathrm{A}$ and $\mathrm{B}$ can be any of the above final destinations. The RF module was placed on convenient locations where the user needs to take a turn or at the locations of interests. The routes and all look ups are stored in an SD card. So what the device does is that it gets the destination from the user and checks the staring and end points, then from the SD card it checks which is the next check point it needs to reach and then directs the user to that location and then to the next till he/she reaches the destination.

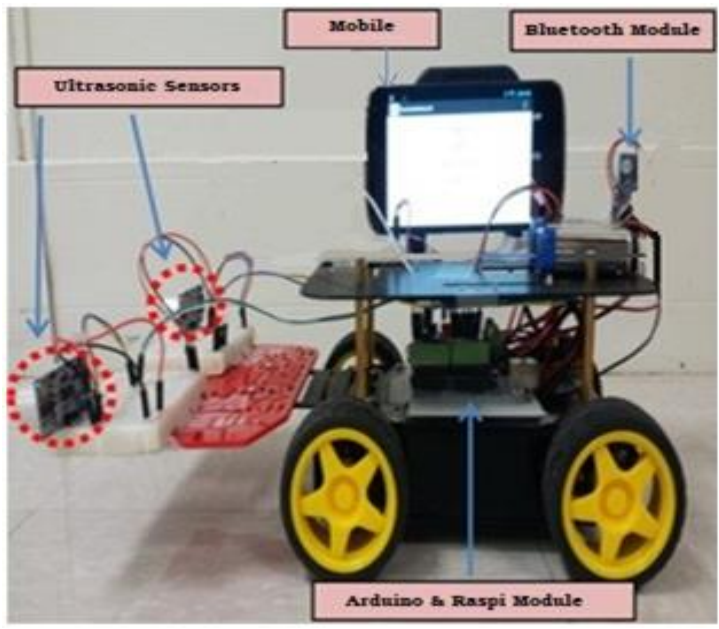

Fig. 1.The device with all components

\section{B. Components and their functions}

The Arduino: is used to control the motion of the rover and to get the data from the mobile device. The HT-05 blue tooth module is used to interface the Arduino with the mobile device, the destination to which the user must travel to will be collected by the mobile device and will be passed on to the Arduino using the blue tooth module. Then the Arduino will send the destination address to raspy. The L293d motor driver IC is used to make the Arduino control the rover. The Arduino gets the information from the raspberry pi about which check point it must move to next. It then uses the digital compass on board to check for the heading. What actually happens is that if the next check point is at 80.25 degree to the users current position then the Arduino will sent data to the L293d to move forward, if the heading changes due to some reason due to the curvature of the road or unevenness of the road then the Arduino corrects the heading accordingly so as the keep the heading in the center.

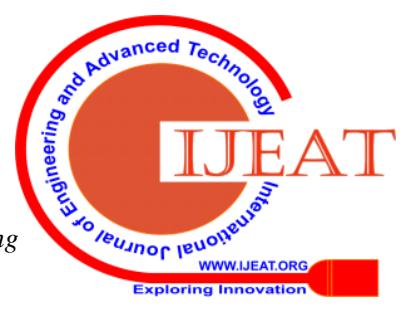


The Arduino is also taking input from the ultrasonic sensors to detect obstacles in front of the device. If an obstacle is encountered it will sent the information to the user and the raspy.

Raspberry Pi Module: The main task of the raspberry pi module is to coordinate the working of all the components in this device to make the best experience for the user. The raspy will get the destination location from the Arduino. And it will receive the camera data from the mobile device. It will use the data from the SD card module to check for the current location and the path it must cover to reach the desired destination. Then what the raspy does is that it gives the Arduino the heading of the current location and also the heading of the next check point it must go to. The next task of the Raspy is to check the data from the RF module if the data from the module is received it cross checks with the stored value and checks which location it reached, if it's the correct location then it stores that as the current location in the SD card and then gives the data of the next check point to the Arduino. If it is at a wrong location a rerouting is done to reach the correct location. The camera of the mobile will be always connected to the raspy, so the input from the camera is constantly monitored to detect faces of people coming towards the user and then if it matches the face of someone from the data base then this information is given to the Arduino and the Arduino will notify the user about the person in front of him. When the Arduino detects an obstacle in front of the user then the information is received by the raspberry $\mathrm{pi}$, then the raspy will use the camera to identify what is the obstacle in front of the user and will notify the user of that obstacle and when user gives the command to re-route it will move forward by avoiding the obstacle.

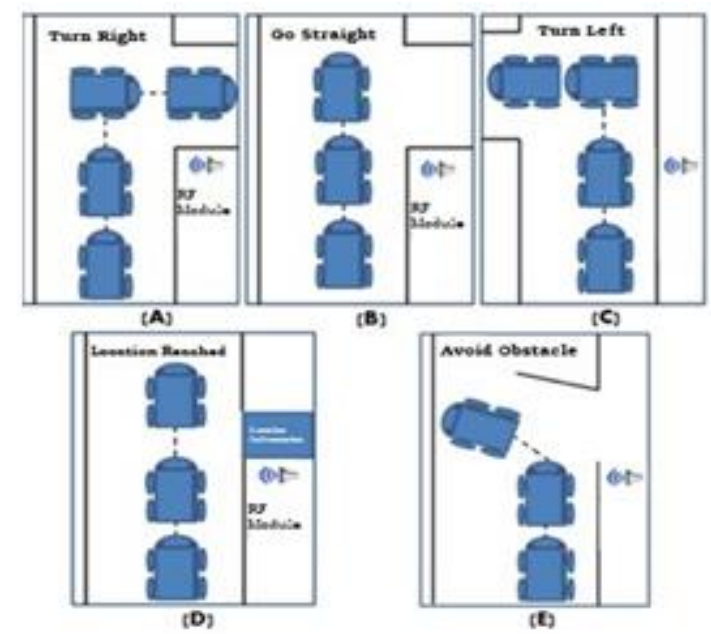

Fig. 2. Routing the device at different check points

The Obstacle detection is done currently using an ultra sonic sensor and a camera. If the ultrasonic sensor senses an obstacle in front of the user on the ground the raspy will initiate the obstacle detection and routing protocol, what happens here is the Raspy will store the next location address and then it instructs the Arduino to calculate the angle of the obstacle with respect to the device. Then Arduino instructs the device to move in that angle till the obstacle is crossed, then the (180-that angle) degree is turned so that the obstacle can be avoided. Now the Arduino is again given the heading of the next check point and normal procedure is continued.

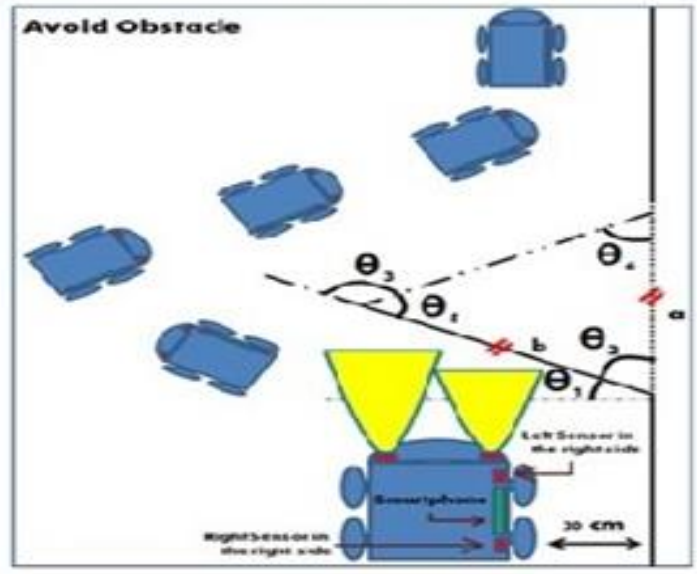

Fig.3. Obstacle avoidance

$$
\begin{aligned}
& \Theta 1=\arctan (D / \text { Dist_sensor }) \\
& \Theta 2=90^{\circ}-\Theta 1 \\
& \Theta 3=180-\Theta 5 \\
& \Theta 4=\Theta 5=(180-\Theta 2) / 2
\end{aligned}
$$

The mobile application is the thing that we use to interface the device to the user, the app was made using MIT App inventor 2. The application can be opened by using the Google assistant. What the user will do is that he will open the application and then shake the phone, this will open a speech input item that allows the user to speak of the location that he needs to travel to, then the device will check in its data base and will ask the user for confirmation, ones the conformation is obtained then the device sends the data to the Arduino using the Bluetooth module. Then the next task of the device is to give the user awareness about the trip, where they have reached, warn the user about any obstacles in front of him. It will also receive information about the person in front of the user and will notify him of the name of person in front of him.

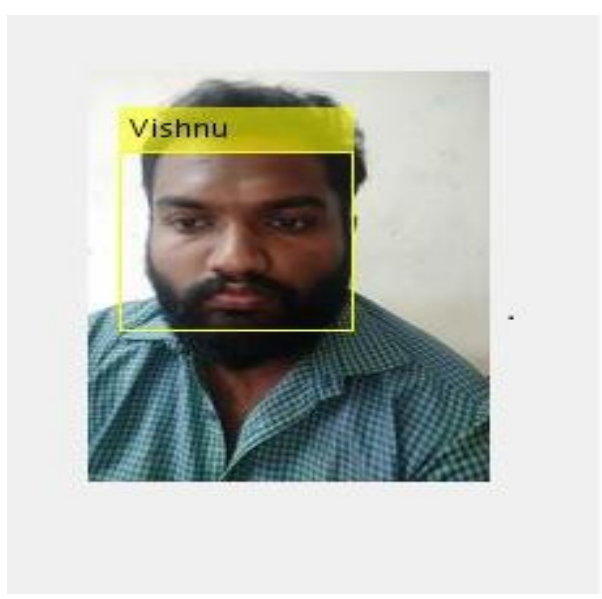

Fig.4. User identification from camera of mobile on Raspberry pi 
The RF module is used for the location identification; these are placed at the check points and the different destination locations. Each RF module is having a specific address assigned to it, the transmitter is placed at the location and the receiver is placed on the rover. When the user reaches the location the receiver will detect the signal from the transmitter and the data transmitted by each transmitter is its address, so the receiver can then pass the address details to the Raspy, which checks if the location is accurate or not. As mentioned earlier before the work began the magnetic heading was identified and stored in the SD card, then the heading through which we have to travel from each location to other was measured using the digital compass and stored. Then the heading was tested by moving the digital compass trough the paths and errors were minimized.

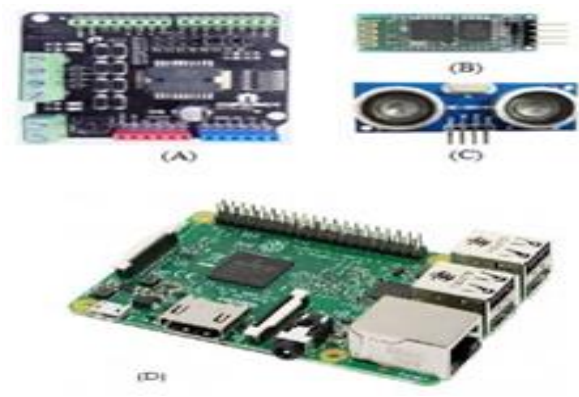

Fig.5. L293D motor drive (B) HC-05 Bluetooth Module (C) Ultrasonic Sensor (D) Raspberry pi 3
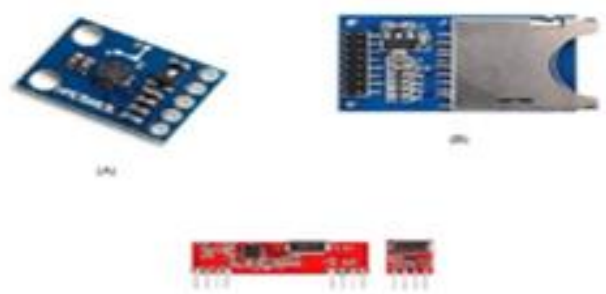

Fig.6. (A) Magnetic Sensor (B) SD Card Module (C) RF Transmitter and Receiver Module

\section{LIMITATIONS AND FUTURE MODIFICATIONS}

The device should be custom made for all individual users and the RF modules should be placed in the correct places, the battery of these modules must be regularly changed or they should be maintained. The routing that we are doing now is custom made for the user according to his preference and location of use. If he is using the device in the University or at his home town we need to route the device accordingly and the RF modules must be placed accordingly and must be maintained. If we use GPS then the routing can be done easily and there won't be the need to customize the path for each users, but the problem with using GPS is that it does not consider if the user is in the side or middle of the road but in our module the device is initially placed near the RF module which are all placed on the right side of the road. If we do not use a custom path the problem is that if we use Google map or other services for routing then the shortest path will be considered for routing if that is done then sometimes the shortest path might not be the safest for a visually impaired person, so this is where the usefulness of a custom path comes in. So the improvement that needs to be worked on is to make the routing algorithm more general purpose, in the present system the magnetic heading of each location is explicitly stored in the SD card and the digital compass reading to be kept stable between two random check points A and $\mathrm{B}$. So if we are giving the magnetic heading of two locations then the program should identify the route to guide the user in so as to reach the destination or the compass reading that the user must maintain to reach the destination.

\section{RESULTS AND DISCUSSIONS}

A device that will guide a visually impaired person to his destination based on a predefined path was made and was successfully tested in our University campus. We did the testing with a visually impaired research scholar in our University and his inputs were carefully monitored. The few improvements that he suggested was that the device was not moving fast enough when he compares to his original travel using his walking stick. He then told us that there were instances when the connection between mobile device and the Arduino using the blue tooth module was broken, the device notified him of the failed connection and he was able to reconnect it easily but the connection was being lost a couple of times. This is a common problem with the HC-05 module and we fixed it by using another Bluetooth module BLE Link Bee. Another problem was in the obstacle detection and routing, here the obstacles will be detected by the ultrasonic sensor and the camera. But the problem is that if the obstacle is a small plank or something that the user can just cross over but the car can't then it was causing a problem. The situation was that while testing there was a tree branch that was on the ground the device notified him of the tree branch but he was not notified of how big a branch it was, actually he could have just crossed it as it was a small branch, but the rover took him sideways around the branch and then moved back to the other side before returning to the old destination. Actually the device was spot on and did exactly what it was supposed to do, but the obstacle in this case was not a very big one or a hurdle that a blind person could find difficulty in crossing. During the obstacle detection mode it informs the user of an obstacle and tells him he is being rerouted, he informed us that if he had used the cane he could have felt the branch and he could have crossed it easily but here what happened was that he was not knowing how big of an obstacle is in front of him. If the blind person were using a cane then he will feel the brick and understand that it is not a big obstacle and he will just cross it, but now it is taking the user around it, So a method that can be adopted to improve the object detection in the camera and to give the user an idea about what type of obstacle is in front of him and how tall that obstacle is. If this can be done then the user can just pick up the device just like he would with a cane and place it on the other side or on top of the brick layer or the obstacle in front of him.

\section{CONCLUSION}

Main objective of any blind navigation system should be to maximize the efficiency of navigation, minimize the effort that the blind person would have to make and to minimize his dependency on a person with the ability to see. It should be cheap, adapt to all environment and should be easy to use.

Blue Eyes Intelligence Engineering

DOI: 10.35940/ijeat.C6284.029320

Journal Website: www.ijeat.org 
In this work a robot assisted routing and navigational system for visually impaired people was designed and tested. The device receives the user's instruction about where the device must guide him/her to and then it will use the on-board circuits to guide the user safely to the desired destination. The device does not cause any inconvenience to the user and will inform the user about the names of pre-saved people in front of him and will also give information about the obstacles in front of him.

\section{REFERENCES}

1. Nicholas A. Giudice Gordon E. Legge, "Blind Navigation and the Role of Technology," The Third International Symposium on Wearable computer, San Francisco CA, pp. 37-43.

2. Jack M. Loomis, Reginald G. Golledge, Roberta L. Klatzky, "Navigation System for the Blind:Auditory Display Modesand

Guidance", Presence: Teleoperators and Virtual Environment,Vol.7, Issue No.2, pp 193-203 by the Massachusetts Institute of Technology, April 1998.

3. J. Wang, "Electronic white cane for blind people navigation Assistance", World Automation Congress 2010 TSI Press. Kobe, Japan pp 90 - 97

4. Vítor Filipe, Filipe Fernandes, Hugo Fernandes, António Sousa, Hugo Paredes, João Barroso,, "Blind navigation support system based on Microsoft Kinect", Proceedings of the 4th International Conference on Software Development for Enhancing Accessibility and Fighting Info-exclusion. Milan, Italy. pp $94-101$

5. Steve Mann, Jason Huang, Ryan Janzen, Raymond Lo, Valmiki Ramprasad Alexander Chen, Taqveer Doha, "Blind Navigation with a Wearable Range Camera and Vibrotactile Helmet", International Conference on Computing Machinery '18, Scottsdale, Arizona, USA. pp 44-49.

6. Mounir Bousbia-Salah, Abdelghani Redjati, Mohamed Fezari, "An ultrasonic navigation system for blind people", IEEE International Conference on Signal Processing and Communications (ICSPC 2007), Dubai, United Arab Emirates, pp 24-27 November 2007,

7. Senjuti Dutta , Mridul S. Barik, Chandreyee Chowdhury, and Deep Gupta, "Divya-Dristi: A Smartphone based Campus Navigation System for the Visually Impaired", The Third International Symposium on Wearable computer, San Francisco, CA, pp. 159-166, 2018

8. Abdelsalam (Sumi) Hela, Steven Edwin Moore, Balaji Ramachandran, "Drishti: An Integrated Navigation System for Visually Impaired and Disabled", Proceedings Fifth International Symposium on Wearable Computers Zurich, Switzerland. Oct, 2001 pp 14-18

9. Apostolos Meliones, Jairo Lozano Llorente, "Study and Development of a Sonar Obstacle Recognition Algorithm for Outdoor Blind Navigation ", Pervasive Technologies Related to Assistive Environments, June 5-7, 2019, Rhodes, Greece Association for Computing Machinery.ACM pp 153-159

10. Milici, Stefano; Esposito, Ambra; Staderini, Enrico M., "A Wireless Indoor navigation Aid for Visually Impaired People using UWB Localization Infrastructure and an Original Wayfinding Algorithm", 14th International Conference on Location Based Services, Zurich, Switzerland, January, 2018 pp 15-17,

11. Ali Khan, Aftab Khan, Muhammad Waleed "Wearable Navigation Assistance System for the Blind and Visually Impaired", International Conference on Innovation and Intelligence for Informatics, Computing, and Sakhier, Bahrain, Bahrain , November, 2018 pp 107-115

12. Masayuki Murata , Dragan Ahmetovic , Daisuke Sato, Hironobu Takagi, Kris M. Kitani, Chieko Asakawa "Smartphone-based Indoor Localization for Blind Navigation across Building Complexes." IEEE International Conference on Pervasive Computing and Communications Athens, Greece March 2018 pp 112-119

13. Andreas Hub Joachim Diepstraten Thomas Ertl, "Design and Development of an Indoor Navigation and Object Identification System for the Blind", ASSETS'04, October 18-20, 2004, Atlanta, Georgia, USA. pp 44-51

14. Sakmongkon Chumkamon, Peranitti Tuvaphanthaphiphat, Phongsak Keeratiwintakorn, “A Blind Navigation System Using RFID for Indoor Environment ", Proceedings of ECTI-CON, Krabi, Thailand, May, 2017. pp 55-63

15. Citation: Lima, A., Mendes, D. and Paiva, S. (2018). "Outdoor Navigation Systems to Promote Urban Mobility to Aid Visually Impaired People." Journal of Information Systems Engineering \& Management, 3(2), 18. Frankfurt, Germany, May 2018

16. Saki Asakawa, João Guerreiro, Daisuke Sato, Hironobu Takagi, Dragan Ahmetovic, Desi Gonzalez, Kris M. Kitani, Chieko Asakawa, "An Independent and Interactive Museum Experience for Blind People"
Procedings of Web for all Personalisation. May, 2019, San Francisco, CA, USA, pp 66-76

17. Pelin Angin, Bharat Bhargava Sumi Helal, "A Mobile-Cloud Collaborative Traffic Lights Detector for Blind Navigation", Eleventh International Conference on Mobile Data Management, Kansas City, MO, USA, May 2010 pp 22-30

18. João Guerreiro, Dragan Ahmetovic, Kris Kitani, Chieko Asakawa, Daisuke Sato, "Airport Accessibility and Navigation Assistance for People with Visual Impairments ”, CHI 2019, Paper 16, May 4-9, 2019 , Glasgow, Scotland, UK pp 77-86

19. Lisa Ran, Sumi Helal and Steve Moore, "Drishti: An Integrated Indoor/Outdoor Blind Navigation System and Service", Proceedings of the Second IEEE Annual Conference on Pervasive Computing and Communications, Orlando, FL, USA, March, 2004 pp 66-70

20. Cole Gleason, Carnegie Mellon Dragan Ahmetovic, Saiph Savage, Carlos Toxtli, Carl Posthuma, Chieko Asakawa, Kris M. Kitani, "Crowd sourcing the Installation and Maintenance of Indoor Localization Infrastructure to Support Blind Navigation", Proceedings of the ACM on Interactive, Mobile, Wearable and Ubiquitous Technologies, Vol. 2, No. 1, Article 9: March 2018

\section{AUTHORS PROFILE}

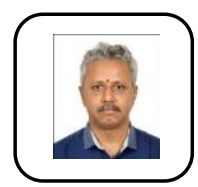

Dr. T. Shanmuganantham, is an Associate Professor of Department of Electronics Engineering, Pondicherry Central University, Pondicherry. He received his B.E. degree in Electronics \& Communication Engineering from University of Madras in the year 1996, M.E. degree in Communication Systems from Madurai Kamaraj University in the year 2000 and received his Ph.D. degree (Received Gold Medal) in the field of Antenna Engineering from National Institute of Technology (NIT), Tiruchirappalli in the year 2010 under the guidance of Emeritus Prof.S.Raghavan, Dept. of Electronics \& Communication Engineering, NIT, Tiruchirappalli. He holds a Google Scholar H-Index of 21 with over 3000 citations, Research Gate with over 30 points and 225 SCOPUS publications as per database also his name listed Top 10 researcher in Pondicherry University. His research interests include Antenna Engineering, Microwave/Millimetre-Wave Engineering and MEMS/NEMS. Specific antenna design for 5G/6G, MIMO, Array antenna for beam forming and beam steering, wireless on body communications, inbody communications (implantable antenna), Automotive, Healthcare, Satellite, Space, and Wireless industries. He has published 207 papers in Referred Journals, 410 papers Conference proceedings and 12 Book Chapters Published in Springer Nature and Elsevier.

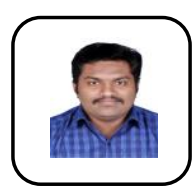

Mr. Vishnu Prasad, S is pursuing M.Tech degree (4thsemester) in the department of Electronics Engineering, School of Engineering and Technology, Pondicherry University, India. He received his B.Tech degree from Amal Jyothi College of Engineering, affiliated to Mahatma Gandhi University Kerala in 2018. He have presented a paper in the International Conference on New Trends in Engineering and Technology, 2018. He is an IEEE member from 2016. 